\title{
Bosonization and Quantum Hydrodynamics
}

\author{
Girish S. Setlur \\ Department of Physics, Indian Institute of Technology, Guwahati, \\ North Guwahati, Assam 781 039, India
}

(Dated: August 1, 2021)

\begin{abstract}
It is shown that it is possible to bosonize fermions in any number of dimensions using the hydrodynamic variables, namely the velocity potential and density. The slow part of the Fermi field is defined irrespective of dimensionality and the commutators of this field with currents and densities are exponentiated using the velocity potential as conjugate to the density. An action in terms of these canonical bosonic variables is proposed that reproduces the correct current and density correlations. This formalism in one dimension is shown to be equivalent to the Tomonaga-Luttinger approach as it leads to the same propagator and exponents. We compute the one-particle properties of a spinless homogeneous Fermi system in two spatial dimensions with long-range gauge interactions and highlight the metal-insulator transition in the system. A general formula for the generating function of density correlations is derived that is valid beyond the random phase approximation. Finally, we write down a formula for the annihilation operator in momentum space directly in terms of number conserving products of Fermi fields.
\end{abstract}

PACS numbers: 71.10.Pm,73.21.Hb,73.23.Ad

\section{INTRODUCTION}

The study of interacting fermions in one spatial dimension has attracted renewed attention in the recent past with the experimental realization of nano-structures and simulation of quasi-one dimensional environments [1]. Many theoretical works that seek to provide a basis for testing fundamental physics, as well as those that propose new scenarios for practical application of these ideas have emerged [2]. All these are based on the Luttinger liquid paradigm of Haldane [3] where homogeneous one dimensional systems are considered to be a specific kind of anomalous metal characterized by power law singularities in the Green function. However, this paradigm is valid mainly for short-range interactions. For a specific kind of long-range interaction $\left(v(q)=2 e^{2} / q^{2}\right)$, one of the authors have shown in an earlier work 4 that the system is characterized by essential singularities rather than power-law singularities. Also, in another work [5], the formal theoretical framework upon which the Tomonaga-Luttinger picture is based, was critiqued. In particular, this approach fails to provide a proper description of the ground state of a one dimensional homogeneous Fermi system interacting via the gauge potential as has been pointed out [4]. That the presence of Klein factors in this approach, renders the analysis of the three-wire junction problematic has been noted by Chamon et al [] $]$ and by Lal et al[2].

In this paper we show that fermions in one and higher dimensions may be conveniently bosonized by exponentiating the slow part of the field using current algebra. In particular, it is shown that no Klein factors need be invoked ${ }^{1}$. The right movers and left movers are shown to obey fermion commutation rules nonetheless. In one dimension, this fact is already known to the experts [8] [9], however in higher dimensions it is probably less well known. Nevertheless, we present the one dimensional formalism even if there is some overlap with established methods, if only to motivate the generalization to higher dimensions. We also express the annihilation operator in momentum space directly in terms of a certain combination of number conserving products of Fermi fields known as sea-bosons $[\underline{5}$ thereby completing the formalism introduced earlier [] . It is worth emphasizing that it is the slow part of the field operator that is easily expressed in terms of the bosons rather than the full field as was implied in one of the authors' earlier work [5]. The present approach, together with the action mentioned in the abstract, can be argued to be quite adequate in studying gauge interactions in higher dimensions as well.

\section{FORMALISM}

For a pedagogical introduction to bosonization in one dimension, there are many reviews available $[9]$. For the sake of brevity, we shall only discuss the important ideas. First we consider fermions in one spatial dimension.

${ }^{1}$ More precisely, just one global Klein factor suffices in all dimensions rather than one for each kind of mover [5], 7]. 
Define the right movers and left movers as usual $\left(\Lambda \ll k_{F}\right.$ is the momentum cutoff around the Fermi points), $\psi_{R}(x)=\frac{1}{\sqrt{L}} \sum_{k} e^{i k x} c_{k_{F}+k} \theta(\Lambda-|k|)$ and $\psi_{L}(x)=\frac{1}{\sqrt{L}} \sum_{k} e^{i k x} c_{-k_{F}+k} \theta(\Lambda-|k|)$. These are slowly varying in space. In this theory we assume that in the end $k_{F} \gg \Lambda \rightarrow \infty$. Now we define the density fluctuation operator and its conjugate as follows ( with $p$ and $q$ unrestricted ), $\rho_{q}=\sum_{p} c_{p+q / 2}^{\dagger} c_{p-q / 2}$ and $X_{q}=\sum_{p}\left(\frac{i p . q}{q^{2} N^{0}}\right) c_{p-q / 2}^{\dagger} c_{p+q / 2}$. These operators obey the following commutation relations,$\left[X_{q}, X_{q^{\prime}}\right]=\left[\rho_{q}, \rho_{q^{\prime}}\right]=0$ and $\left[X_{q}, \rho_{q^{\prime}}\right]=i \delta_{q, q^{\prime}}$ 5]. Since only terms with small $q$ contribute ( the $\psi$ 's are slowly varying ) we have, $\left[\psi_{R}(x), \rho_{q}\right]=e^{i q x} \psi_{R}(x),\left[\psi_{R}(x), X_{q}\right]=e^{-i q x}\left(\frac{i \pi}{q L}\right) \psi_{R}(x)$ and $\left[\psi_{L}(x), \rho_{q}\right]=e^{i q x} \psi_{L}(x),\left[\psi_{L}(x), X_{q}\right]=-e^{-i q x}\left(\frac{i \pi}{q L}\right) \psi_{L}(x)$. These may be exponentiated as follows.

$$
\begin{gathered}
\psi_{R}(x)=e^{-i \sum_{q} e^{i q x} X_{q}} e^{-\pi \sum_{q} \frac{e^{-i q x}}{q L} \rho_{q}} \sqrt{\frac{\Lambda}{2 \pi}} \\
\psi_{L}(x)=e^{-i \sum_{q} e^{i q x} X_{q}} e^{\pi \sum_{q} \frac{e^{-i q x}}{q L} \rho_{q}} \sqrt{\frac{\Lambda}{2 \pi}}
\end{gathered}
$$

The multiplicative factor, namely $\sqrt{\frac{\Lambda}{2 \pi}}$, comes from the observation that, $\left\langle\psi_{R}^{\dagger}(x) \psi_{R}(x)\right\rangle=\left\langle\psi_{L}^{\dagger}(x) \psi_{L}(x)\right\rangle=\frac{\Lambda}{2 \pi}$. The Eq.(1) and Eq.(2) obey fermion commutation rules without the need for Klein factors for $x \neq x^{\prime}$ which is easy to verify. In the usual approach to bosonization, these formulae are regularized by introducing a decaying exponential[9]. The regularization is needed to reproduce the delta function in the fermion commutation rules, $\left\{\psi_{R / L}(x), \psi_{R / L}^{\dagger}\left(x^{\prime}\right)\right\}=\delta\left(x-x^{\prime}\right)$. Here too, we adopt a similar but not quite the same approach. In this regard we postulate,

$$
\begin{aligned}
& \psi_{R}(x)=e^{-i \sum_{q} e^{i q x} X_{q}} e^{-\pi \sum_{q} \frac{e^{-i q x}}{q L} \rho_{q}}\left(\frac{\Lambda}{2 \pi}+\frac{1}{L} \sum_{q \neq 0} e^{-i q x}\left[\frac{1}{2} \rho_{q}+\frac{i q L}{2 \pi} X_{-q}\right]\right)^{\frac{1}{2}} \\
& \psi_{L}(x)=e^{-i \sum_{q} e^{i q x} X_{q}} e^{\pi \sum_{q} \frac{e^{-i q x}}{q L} \rho_{q}}\left(\frac{\Lambda}{2 \pi}+\frac{1}{L} \sum_{q \neq 0} e^{-i q x}\left[\frac{1}{2} \rho_{q}-\frac{i q L}{2 \pi} X_{-q}\right]\right)^{\frac{1}{2}}
\end{aligned}
$$

That is, we add the density fluctuations of right/left movers to the (nearly infinite) mean density. This means that the commutation relations with $\rho_{q}$ and $X_{q}$ are now going to be invalid but the corrections are inverse in the number of right and left movers. Since $\Lambda \rightarrow \infty$ we may write,

$$
\begin{aligned}
& \psi_{R}(x)=e^{-i \sum_{q \neq 0} e^{i q x} X_{q}} e^{-\pi \sum_{q \neq 0} \frac{e^{-i q x}}{(q-i \epsilon) L} \rho_{q}} e^{\frac{\pi}{L \Lambda} \sum_{q \neq 0} e^{-i q x} e^{-|q| / \Lambda}\left[\frac{1}{2} \rho_{q}+\frac{i q L}{2 \pi} X_{-q}\right]}\left(\frac{\Lambda}{2 \pi}\right)^{\frac{1}{2}} \\
& \psi_{L}(x)=e^{-i \sum_{q \neq 0} e^{i q x} X_{q}} e^{\pi \sum_{q \neq 0} \frac{e^{-i q x}}{(q-i \epsilon) L} \rho_{q}} e^{\frac{\pi}{\Lambda L} \sum_{q \neq 0} e^{-i q x} e^{-|q| / \Lambda}\left[\frac{1}{2} \rho_{q}-\frac{i q L}{2 \pi} X_{-q}\right]}\left(\frac{\Lambda}{2 \pi}\right)^{\frac{1}{2}}
\end{aligned}
$$

where $\epsilon \rightarrow 0^{+}$. It can be shown that Eq.(5) and Eq.(6) obey the following commutation relations,

$$
\begin{gathered}
\left\{\psi_{R}(x), \psi_{L}\left(x^{\prime}\right)\right\}=\left\{\psi_{R}(x), \psi_{L}^{\dagger}\left(x^{\prime}\right)\right\}=\left\{\psi_{R}(x), \psi_{R}\left(x^{\prime}\right)\right\}=\left\{\psi_{L}(x), \psi_{L}\left(x^{\prime}\right)\right\}=0 \\
\left\{\psi_{R}(x), \psi_{R}^{\dagger}\left(x^{\prime}\right)\right\}=\left\{\psi_{L}(x), \psi_{L}^{\dagger}\left(x^{\prime}\right)\right\}=\delta\left(x-x^{\prime}\right)
\end{gathered}
$$

These nuances are not very important for the practical computations. In fact, we may use the unregularized fields for computing the propagators with finite spatial and temporal separations with impunity. We remarked that there are no Klein factors in the formalism. This refers to the need to invoke two different Klein factors, one for each kind of mover, in the usual approach [9]. In the present approach, there has to be an overall global Klein factor namely $X_{q=0}$ that was defined in our earlier work [7]. We may now compute the propagators as follows. It can be shown that for 
the free Fermi theory, $\left\langle\psi_{R}^{\dagger}\left(x^{\prime}, t^{\prime}\right) \psi_{R}(x, t)\right\rangle \sim \frac{1}{\left(x-x^{\prime}\right)-v_{F}\left(t-t^{\prime}\right)}$. One may also similarly study the interacting system with equal ease and obtain the right exponents for the Luttinger model.

Next, we wish to generalize these ideas to higher dimensions. This is likely to be complicated since there is nothing called a right mover or left mover in two and more dimensions because the Fermi surface is no longer made of two points but rather, an uncountable infinity of them. The usual approach of Luther and Haldane 3 breaks up the Fermi surface into patches where the separation between the patches $\Delta k_{F} \gg \Lambda \sim \Delta q$. Not only is this contrived, it involves the introduction of one Klein factor in every radial direction. Here we show that there is no need for Klein factors at all (apart from the global one $7 \mid$ ). To this end we have to make the following analogy. Consider the following operators in one dimension, $\psi_{\text {slow }}(x)=\frac{1}{\sqrt{L}} \sum_{k} e^{i\left(k-k_{F} \operatorname{sgn}(k)\right) x} c_{k} \theta\left(\Lambda-|| k\left|-k_{F}\right|\right)$ and $\psi_{\text {diff }}(x)=\frac{1}{\sqrt{L}} \sum_{k} e^{i\left(k-k_{F} \operatorname{sgn}(k)\right) x} c_{k} \operatorname{sgn}(k) \theta\left(\Lambda-|| k\left|-k_{F}\right|\right)$. These are linear combinations of right and left movers which can be obtained from,

$$
\psi(x, y)=\frac{1}{\sqrt{L}} \sum_{k} e^{i\left(k-k_{F} \operatorname{sgn}(k)\right) x} e^{i s g n(k) y} c_{k} \theta\left(\Lambda-|| k\left|-k_{F}\right|\right)
$$

as $\psi_{\text {slow }}(x)=\psi(x, 0) ; \psi_{\text {diff }}(x)=-i \psi_{y}(x, 0)$. By analogy we may generalize these ideas to more than one dimension as follows. We make the following prescription: $\operatorname{sgn}(k) \rightarrow \hat{k}$. Then,

$$
\psi(\mathbf{x}, \mathbf{y})=\frac{1}{\sqrt{V}} \sum_{\mathbf{k}} e^{i\left(\mathbf{k}-k_{F} \hat{k}\right) \cdot \mathbf{x}} e^{i \hat{k} \cdot \mathbf{y}} c_{\mathbf{k}} \theta\left(\Lambda-|| \mathbf{k}\left|-k_{F}\right|\right)
$$

First, this object is a slowly varying function of $\mathbf{x}$. In one dimension, a drastic simplification is possible which is not available in more than one dimension, namely, $e^{i s g n(k) y}=\theta(k) e^{i y}+\theta(-k) e^{-i y}$. In more than one dimension, all possible directions $\hat{k}$ are involved. It is this $\psi(\mathbf{x}, \mathbf{y})$ which is a slowly varying function of $\mathbf{x}$ that is easily expressed in terms of the currents and densities rather than the full $\psi(\mathbf{x})$. This field $\psi(\mathbf{x}, \mathbf{y})$ is the higher dimensional analog of the one dimensional version where only two directions are involved and would correspond to linear combinations of right and left movers. Since this is a slowly varying function of $\mathbf{x}$ we can expect this to involve only $\rho_{\mathbf{q}}$ and $X_{\mathbf{q}}$ for small $\mathbf{q}$ as is the case in one dimension. Define $\rho_{\mathbf{q}}$ and $X_{\mathbf{q}}$ as in Ref. [5]. Then we have,

$$
\begin{gathered}
{\left[\psi(\mathbf{x}, \mathbf{y}), \rho_{\mathbf{q}}\right]=\frac{1}{\sqrt{V}} \sum_{\mathbf{k}} e^{i\left(\mathbf{k}-k_{F} \hat{k}\right) \cdot \mathbf{x}} e^{i \hat{k} \cdot \mathbf{y}} c_{\mathbf{k}-\mathbf{q}} \theta\left(\Lambda-|| \mathbf{k}\left|-k_{F}\right|\right) \approx e^{-i\left(\nabla_{\mathbf{y}} \cdot \mathbf{q}\right)\left(\nabla_{\mathbf{y}} \cdot \mathbf{x}\right)} \psi(\mathbf{x}, \mathbf{y})} \\
{\left[\psi(\mathbf{x}, \mathbf{y}), X_{\mathbf{q}}\right]=\frac{1}{\sqrt{V}} \sum_{\mathbf{k}} e^{i\left(\mathbf{k}-k_{F} \hat{k}\right) \cdot \mathbf{x}} e^{i \hat{k} \cdot \mathbf{y}}\left(\frac{i \mathbf{p} \cdot \mathbf{q}}{N^{0} \mathbf{q}^{2}}\right) \delta_{\mathbf{k}, \mathbf{p}-\mathbf{q} / 2} c_{\mathbf{p}+\mathbf{q} / 2} \theta\left(\Lambda-\| \mathbf{k}\left|-k_{F}\right|\right) \approx e^{i\left(\nabla_{\mathbf{y}} \cdot \mathbf{q}\right)\left(\nabla_{\mathbf{y}} \cdot \mathbf{x}\right)} \frac{k_{F} \mathbf{q} \cdot \nabla_{\mathbf{y}}}{N^{0} \mathbf{q}^{2}} \psi(\mathbf{x}, \mathbf{y})}
\end{gathered}
$$

These two rules may be exponentiated as follows.

$$
\psi(\mathbf{x}, \mathbf{y})=e^{-i \sum_{\mathbf{q}} e^{-i\left(\nabla_{\mathbf{y}} \cdot \mathbf{q}\right)\left(\nabla_{\mathbf{y}} \cdot \mathbf{x}\right)} X_{\mathbf{q}}} e^{i \sum_{\mathbf{q}} e^{i\left(\nabla_{\mathbf{y}} \cdot \mathbf{q}\right)\left(\nabla_{\mathbf{y}} \cdot \mathbf{x}\right)}\left(\frac{k_{F} \mathbf{q} \cdot \nabla_{\mathbf{y}}}{N^{0} \mathbf{q}^{2}}\right) \rho_{\mathbf{q}}} F(\mathbf{x}, \mathbf{y})
$$

Here $F(\mathbf{x}, \mathbf{y})$ is an integration constant independent of both $X_{\mathbf{q}}$ and $\rho_{\mathbf{q}}$. It obeys the constraint that $\nabla_{\mathbf{y}}^{2} F(\mathbf{x}, \mathbf{y})=$ $-F(\mathbf{x}, \mathbf{y})$ since the unit vector $\hat{k}$ multiplies $\mathbf{y}$. In practical computations, one computes the propagator of the interacting theory using the prescription in Eq.113) and multiplies and divides by the propagator of the free theory and in the division uses the one obtained from Eq.(13) and in the numerator uses the one obtained from elementary considerations. This eliminates $F(\mathbf{x}, \mathbf{y})$ and a closed expression for the full dynamical (albeit slow part) of the Green function may be written down in terms of current-current, current-density, and density-density correlations. The full propagator may be related to the current and density correlations as follows.

$$
\begin{gathered}
\left\langle\psi^{\dagger}\left(\mathbf{x}^{\prime}, \mathbf{y}^{\prime}, t^{\prime}\right) \psi(\mathbf{x}, \mathbf{y}, t)\right\rangle=e^{\frac{1}{2} \sum_{\mathbf{q}}\left(\frac{k_{F} \mathbf{q} \cdot \nabla_{\mathbf{y}^{\prime}}}{N^{0} \mathbf{q}^{2}}\right)^{2} \ll \rho_{-\mathbf{q}}\left(t^{\prime}\right) \rho_{\mathbf{q}}\left(t^{\prime}\right) \gg} e^{\frac{1}{2} \sum_{\mathbf{q}}\left(\frac{k_{F} \mathbf{q} \cdot \nabla_{\mathbf{y}}}{N^{0} \mathbf{q}^{2}}\right)^{2} \ll \rho_{-\mathbf{q}}(t) \rho_{\mathbf{q}}(t) \gg} \\
e^{-\sum_{\mathbf{q}} \ll X_{-\mathbf{q}}\left(t^{\prime}\right) X_{\mathbf{q}}\left(t^{\prime}\right) \gg} e^{-\sum_{\mathbf{q}} e^{i\left(\nabla_{\mathbf{y}} \cdot \mathbf{q}\right)\left(\nabla_{\mathbf{y}} \cdot \mathbf{x}\right)} e^{-i\left(\nabla_{\mathbf{y}^{\prime}} \cdot \mathbf{q}\right)\left(\nabla_{\mathbf{y}^{\prime}} \cdot \mathbf{x}^{\prime}\right)}\left(\frac{k_{F} \mathbf{q} \cdot \nabla_{\mathbf{y}^{\prime}}}{N^{0} \mathbf{q}^{2}}\right) \ll \rho_{-\mathbf{q}}\left(t^{\prime}\right) X_{-\mathbf{q}}(t) \gg} \\
e^{\sum_{\mathbf{q}} e^{-i\left(\nabla_{\mathbf{y}} \cdot \mathbf{q}\right)\left(\nabla_{\mathbf{y}} \cdot \mathbf{x}\right)} e^{i\left(\nabla_{\mathbf{y}^{\prime}} \cdot \mathbf{q}\right)\left(\nabla_{\mathbf{y}^{\prime}} \cdot \mathbf{x}^{\prime}\right)}\left(\frac{k_{F} \mathbf{q} \cdot \nabla_{\mathbf{y}}}{N^{0} \mathbf{q}^{2}}\right) \ll X_{-\mathbf{q}}\left(t^{\prime}\right) \rho_{-\mathbf{q}}(t) \gg} e^{\sum_{\mathbf{q}} e^{-i\left(\nabla_{\mathbf{y}^{\prime}} \cdot \mathbf{q}\right)\left(\nabla_{\mathbf{y}^{\prime}} \cdot \mathbf{x}^{\prime}\right)} e^{i\left(\nabla_{\mathbf{y}} \cdot \mathbf{q}\right)\left(\nabla_{\mathbf{y}} \cdot \mathbf{x}\right)}\left(\frac{k_{F} \mathbf{q} \cdot \nabla_{\mathbf{y}}}{N^{0} \mathbf{q}^{2}}\right)\left(\frac{k_{F} \mathbf{q} \cdot \nabla_{\mathbf{y}^{\prime}}}{N^{0} \mathbf{q}^{2}}\right) \ll \rho_{-\mathbf{q}}\left(t^{\prime}\right) \rho_{\mathbf{q}}(t) \gg}
\end{gathered}
$$




$$
e^{\sum_{\mathbf{q}} e^{i\left(\nabla_{\mathbf{y}^{\prime}} \cdot \mathbf{q}\right)\left(\nabla_{\mathbf{y}^{\prime}} \cdot \mathbf{x}^{\prime}\right)} e^{-i\left(\nabla_{\mathbf{y}} \cdot \mathbf{q}\right)\left(\nabla_{\mathbf{y}} \cdot \mathbf{x}\right)} \ll X_{-\mathbf{q}}\left(t^{\prime}\right) X_{\mathbf{q}}(t) \gg}\left\langle\psi^{\dagger}\left(\mathbf{x}^{\prime}, \mathbf{y}^{\prime}, t^{\prime}\right) \psi(\mathbf{x}, \mathbf{y}, t)\right\rangle_{0}
$$

where $\ll \ldots \gg=<\ldots>-<\ldots\rangle_{0}$ and the subscript $\left.<\ldots\right\rangle_{0}$ refers to the quantities for the noninteracting system. Next we wish to relate the fundamental field $\psi(\mathbf{x})$ to the slow field $\psi(\mathbf{x}, \mathbf{y})$. This exercise is essential in order to ensure a logical progression of ideas, also, it is the Green function of the fundamental field that is of physical significance. To accomplish this we may invert Eq.(10) as follows.

$$
\int \frac{d \mathbf{x}}{V} e^{-i w \hat{p} . \mathbf{x}} \int_{v_{0}} \frac{d \mathbf{y}}{v_{0}} e^{-i \hat{p} \cdot \mathbf{y}} \psi(\mathbf{x}, \mathbf{y})=\frac{1}{\sqrt{V}} c_{\left(k_{F}+w\right) \hat{p}} \theta(\Lambda-|w|)
$$

Since the vector $\mathbf{y}$ is dimensionless, we have to have an arbitrary (formally infinite $\sim\left(k_{F} L\right)^{d}$ ) dimensionless 'volume' $v_{0}$ to ensure that the transforms with respect to $\mathbf{y}$ are properly inverted. Thus the fundamental field is,

$$
\psi(\mathbf{X})=\sum_{\mathbf{p}} \int \frac{d \mathbf{x}}{V} e^{i \mathbf{p} \cdot \mathbf{X}} e^{-i\left(|\mathbf{p}|-k_{F}\right) \hat{p} \cdot \mathbf{x}} \int_{v_{0}} \frac{d \mathbf{y}}{v_{0}} e^{-i \hat{p} \cdot \mathbf{y}} \psi(\mathbf{x}, \mathbf{y})=\frac{1}{v_{0}} \int d \mathbf{x} \int_{v_{0}} d \mathbf{y} E(\mathbf{x}, \mathbf{y} ; \mathbf{X}) \psi(\mathbf{x}, \mathbf{y})
$$

where $E(\mathbf{x}, \mathbf{y} ; \mathbf{X})=\frac{1}{V} \sum_{\mathbf{p}} e^{i \mathbf{p} \cdot \mathbf{X}} e^{-i\left(p-k_{F}\right) \hat{p} \cdot \mathbf{x}} e^{-i \hat{p} . \mathbf{y}}$. In one and three spatial dimensions we have respectively,

$$
E(x, y ; X) \approx 2 \cos \left(k_{F} X-y\right) \delta(X-x) ; E(\mathbf{x}, \mathbf{y} ; \mathbf{X}) \approx \frac{k_{F}^{2}}{2 \pi} \frac{\cos \left(\frac{\left(k_{F} \mathbf{X}-\mathbf{y}\right) \cdot(\mathbf{X}-\mathbf{x})}{|\mathbf{X}-\mathbf{x}|}\right)}{|\mathbf{X}-\mathbf{x}|}
$$

In order for the one dimensional version of Eq.(16) to be consistent with Eq.(11) and Eq.(2) we have to ensure that $F(x, y)=\sqrt{\frac{2 \Lambda}{\pi}} \cos (y)$. One may now write down the full propagator of the fundamental field in terms of current and density correlations by replacing the right hand side of the equation below by the expression for the slow propagator in Eq.(14) as follows.

$$
<\psi^{\dagger}(\mathbf{X}, t) \psi\left(\mathbf{X}^{\prime}, t^{\prime}\right)>=\frac{1}{v_{0}^{2}} \int d \mathbf{x}^{\prime} \int_{v_{0}} d \mathbf{y}^{\prime} E\left(\mathbf{x}^{\prime}, \mathbf{y}^{\prime} ; \mathbf{X}^{\prime}\right) \int d \mathbf{x} \int_{v_{0}} d \mathbf{y} E(\mathbf{x}, \mathbf{y} ; \mathbf{X})<\psi^{\dagger}(\mathbf{x}, \mathbf{y}, t) \psi\left(\mathbf{x}^{\prime}, \mathbf{y}^{\prime}, t^{\prime}\right)>
$$

We shall not dwell on this any further, for example, as a nontrivial application, we could calculate the full dynamical propagator of a two dimensional electron gas with long range interactions $(V(r)=\log (r))$. This is bound to lead to the conclusion that the system is a Luttinger liquid with a characteristic exponent, a result that will be more easily and elegantly shown using sea-bosons later on in this article. The current and density correlations may be conveniently calculated [at least at the RPA (random phase approximation) level for translationally invariant systems] using the Lagrangian approach that is conducive to the introduction of gauge fields 10. To this end we derive an action functional for the free electron system in terms of the hydrodynamic variables in any number of dimensions at the RPA level using the sea-boson formalism. Then we argue using general methods, that the functional form of the general action should be similar to the RPA result. First, the derivation of the RPA form of the action in terms of hydrodynamic variables. We borrow the definition of the hydrodynamic variables in terms of sea-bosons from our early work [5].

$$
X_{\mathbf{q}}=\frac{i}{2 N_{0} \epsilon_{\mathbf{q}}} \sum_{\mathbf{k}} \frac{\mathbf{k} \cdot \mathbf{q}}{m}\left[A_{\mathbf{k}}(\mathbf{q})+A_{\mathbf{k}}^{\dagger}(-\mathbf{q})\right] ; \rho_{\mathbf{q}}=\sum_{\mathbf{k}}\left[A_{\mathbf{k}}(-\mathbf{q})+A_{\mathbf{k}}^{\dagger}(\mathbf{q})\right]
$$

We invert these using smearing functions. Let us postulate,

$$
A_{\mathbf{k}}(\mathbf{q})=-i \Gamma_{X}(\mathbf{k}, \mathbf{q}) X_{\mathbf{q}}+\Gamma_{\rho}(\mathbf{k}, \mathbf{q}) \rho_{-\mathbf{q}}
$$

The above is meant to be valid in an average sense and is not an operator identity. If we set,

$$
\Gamma_{X}(\mathbf{k}, \mathbf{q})=\left[A_{\mathbf{k}}(\mathbf{q}), A_{\mathbf{k}}^{\dagger}(\mathbf{q})\right] ; \Gamma_{\rho}(\mathbf{k}, \mathbf{q})=\frac{1}{2 N_{0} \epsilon_{\mathbf{q}}} \frac{\mathbf{k} \cdot \mathbf{q}}{m}\left[A_{\mathbf{k}}(\mathbf{q}), A_{\mathbf{k}}^{\dagger}(\mathbf{q})\right]
$$

then substituting Eq.(20) into Eq.(19) leads to an identity. We know from the sea-boson theory that the hamiltonian of the free Fermi theory is $H=\sum_{\mathbf{k q}} \frac{\mathbf{k} \cdot \mathbf{q}}{m} A_{\mathbf{k}}^{\dagger}(\mathbf{q}) A_{\mathbf{k}}(\mathbf{q})$. Thus the action in terms of sea-bosons is,

$$
S=\int_{0}^{-i \beta} d t \sum_{\mathbf{k q}} A_{\mathbf{k}}^{\dagger}(\mathbf{q})\left(i \partial_{t}-\frac{\mathbf{k} \cdot \mathbf{q}}{m}\right) A_{\mathbf{k}}(\mathbf{q})
$$


Using Eq.(20) in Eq.(22) we find,

$S=\int_{0}^{-i \beta} d t \sum_{\mathbf{k q}}\left[2 \Gamma_{\rho}(\mathbf{k}, \mathbf{q}) \Gamma_{X}(\mathbf{k}, \mathbf{q})\right] \rho_{\mathbf{q}} \partial_{t} X_{\mathbf{q}}-\int_{0}^{-i \beta} d t \sum_{\mathbf{k q}} \Gamma_{\rho}(\mathbf{k}, \mathbf{q})^{2}\left(\frac{\mathbf{k} \cdot \mathbf{q}}{m}\right) \rho_{\mathbf{q}} \rho_{-\mathbf{q}}-\int_{0}^{-i \beta} d t \sum_{\mathbf{k q}} \Gamma_{X}(\mathbf{k}, \mathbf{q})^{2}\left(\frac{\mathbf{k} \cdot \mathbf{q}}{m}\right) X_{\mathbf{q}} X_{-\mathbf{q}}$

This may be simplified to yield,

$$
S=\int_{0}^{-i \beta} d t \sum_{\mathbf{q}} \rho_{\mathbf{q}} \partial_{t} X_{\mathbf{q}}-\int_{0}^{-i \beta} d t \sum_{\mathbf{q}} \frac{\epsilon_{0}}{N_{0}} \rho_{\mathbf{q}} \rho_{-\mathbf{q}}-\int_{0}^{-i \beta} d t \sum_{\mathbf{q}} N_{0} \epsilon_{\mathbf{q}} X_{\mathbf{q}} X_{-\mathbf{q}}
$$

where $\epsilon_{0}=\sum_{\mathbf{k}} \frac{3}{4 N_{0} \epsilon_{\mathbf{q}}} n_{F}(\mathbf{k})\left(\frac{\mathbf{k} \cdot \mathbf{q}}{m}\right)^{2}$. In one dimension $\epsilon_{0}=\frac{k_{F}^{2}}{2 m}$. It appears that we have to use the one dimensional version of the energy scale $\epsilon_{0}$ in higher dimensions as well to reproduce the right static structure factor. The reason for this is similar to the argument that allows Schotte and Schotte 11] to use the Tomonga-Luttinger theory to analyze $\mathrm{X}$-ray absorption in bulk metals in three dimensions (only the s-wave contributes). We now argue that this action is the RPA limit of a more general formula for the action in terms of hydrodynamic variables that will be postulated rather than derived. The claim is that the general action for the free Fermi theory is given in terms of the current and density variables as follows.

$$
S=\int\left(\rho \partial_{t} \Pi-V_{F}([\rho] ; \mathbf{x})-\frac{\rho(\nabla \Pi)^{2}+\frac{(\nabla \rho)^{2}}{4 \rho}}{2 m}\right)
$$

Here $\rho$ and $\Pi$ are conjugate variables and $\mathbf{J}(\mathbf{x}, t)=-\rho(\mathbf{x}, t) \nabla \Pi(\mathbf{x}, t)$ is the current. Also $\rho(\mathbf{x}, t)=$ $\frac{1}{V} \sum_{\mathbf{q} n} e^{-i \mathbf{q} \cdot \mathbf{x}} e^{-z_{n} t} \rho_{\mathbf{q} n}$ and $\Pi(\mathbf{x}, t)=\sum_{\mathbf{q} n} e^{i \mathbf{q} \cdot \mathbf{x}} e^{z_{n} t} X_{\mathbf{q} n}$ where $z_{n}=2 \pi n / \beta$ is the bosonic Matsubara frequency and $\int \equiv \int_{0}^{-i \beta} d t \int d^{d} x$. Here $V_{F}([\rho] ; \mathbf{x})$ is a suitable functional of the density chosen so as to reproduce the properties of the free Fermi theory. To motivate the introduction of this new formalism we point out that in an earlier work [5] one of the authors had argued that the fermionic field (operator) may be expressed in terms of the conjugate variable as follows.

$$
\psi(\mathbf{x}, t)=e^{i \Lambda([\rho] ; \mathbf{x}, t)} e^{-i \Pi(\mathbf{x}, t)} \sqrt{\rho(\mathbf{x}, t)}
$$

This means that the current operator may be written as follows $\mathbf{J}(\mathbf{x}, t)=-\rho(\mathbf{x}, t) \nabla \Pi(\mathbf{x}, t)+\mathbf{C}([\rho] ; \mathbf{x}, t)$, where $\mathbf{C}$ depends on the $\Lambda$ above. Such a general correspondence has already been suggested in the pioneering investigations of Rajagopal and Grest [12]. An examination of the commutator $\left[\mathbf{J}_{i}(\mathbf{x}, t), \mathbf{J}_{j}\left(\mathbf{x}^{\prime}, t\right)\right]$ in terms of the Fermi fields shows that it is expressible as linear combinations of terms involving the currents themselves. By imposing this requirement on the bosonic representation for the current, we find that $\mathbf{C} \equiv 0$. This in turn means that $\Lambda$ should be independent of $\mathbf{x}$ in order for current algebra to be respected. Unfortunately this conflicts with the requirement that $\Lambda$ obey a certain recursion relation that was introduced in an earlier work [5]. Thus we take the point of view that $\Lambda$ depends weakly on $\mathbf{x}$ so that the current is still given by $\mathbf{J}=-\rho \nabla \Pi$. However, the crucial $\Lambda$ makes its appearance in the action as has been depicted in Eq.(25). The viewpoint advocated in this article is that we shall not be too insistent on verifying fermion commutation rules, rather this issue may be cleverly circumvented by forcing agreement with the correlation functions of the free theory and also by ensuring that the commutation rules of Fermi bilinears which are simpler, are recovered properly. Since $V_{F}$ is uniquely determined by this approach, we may work backwards and determine $\Lambda$ and then see if fermion commutation rules are indeed being obeyed. This is likely to be a formidable task and is therefore omitted. There are some subtleties associated with making the transition from the Hamiltonian description to the Lagrangian description. The principal of the these is the question of normal ordering. We note that the action in Eq.(22) is properly normal ordered. The hydrodynamic variables in Eq. (26) have to be expanded in terms of the sea-bosons and terms have to be rearranged so that all the annihilation operators are to the extreme right. Then one de-quantizes Eq.(26) by demoting the various operators to c-number functions before using it to derive the action. The claim is that the resulting ordinary complex number valued function 'simulates' a Grassmannvalued field. The anticommuting property of the Grassmann variable is captured at the level of the propagator by the KMS(Kubo-Martin-Schwinger)-like boundary condition obeyed by the global Klein factor introduced in an earlier work [7]. As it is clear that normal ordering merely redefines $\Lambda$, the actual nature of the function $\Lambda$ is determined by making contact with the free Fermi theory. To this end, we may expand the action in Eq. (25) in powers of density fluctuations. Retaining only the harmonic terms amounts to using the RPA. At this level we may write,

$$
\Lambda([\rho] ; \mathbf{x}, t) \approx \sum_{\mathbf{q} \neq 0, n \neq 0} e^{i \mathbf{q} \cdot \mathbf{x}} e^{z_{n} t} \Lambda_{\mathbf{q} n} \rho_{-\mathbf{q},-n}
$$


where $\Lambda_{\mathbf{q} n}=\frac{\epsilon_{F}}{z_{n} N^{0}}$ and $N^{0}$ is the total number of electrons and $\epsilon_{F}$ is the Fermi energy. Therefore $V_{F}([\rho] ; \mathbf{x})=\rho \partial_{t} \Lambda$. It can be shown that the action in Eq.(25) reproduces the right current and density correlations of the free Fermi theory at the RPA level provided we choose $\Lambda$ to be given by Eq.(27). In particular the choice in Eq.(27) substituted into Eq.(25) yields the RPA level action Eq.(24) derived earlier rigorously using sea-bosons. One may then contemplate adding more terms to this action such as density-density interactions, density-phonon interactions or current densityvector potential interactions to the action and expect to obtain nontrivial results for the one-particle properties. We may also make Eq.(25) plausible by pointing out that it implies a connection between the generating function of density correlations and current correlations. This is relegated to the appendix.

In fact, we may go further and derive a general expression for the generating function of density-density correlations of interacting Fermi systems in terms of the corresponding correlation functions of the free Fermi theory. This exercise is straightforward to perform mainly because the action functional in Eq.(25) has a simple dependence on the conjugate variable $\Pi$ which may be integrated out. To this end, we may define the generating function for the density-density correlation functions as,

$$
Z([U])=\int D[\rho] \int D[\Pi] e^{i S} e^{\int_{0}^{-i \beta} \int d^{d} x U(\mathbf{x}, t) \rho(\mathbf{x}, t)}
$$

Correlation functions involving only the density variable may be obtained as appropriate function derivatives with respect to the source $U$, for example, $<T\left[\rho(\mathbf{x}, t) \rho\left(\mathbf{x}^{\prime}, t^{\prime}\right)\right]>=\delta^{2} Z([U]) /\left.\delta U(\mathbf{x}, t) \delta U\left(\mathbf{x}^{\prime}, t^{\prime}\right)\right|_{U \equiv 0}$. The equation above ( Eq.(28) ) may be formally inverted and an expression for the action with the conjugate variable integrated out, may be written down in terms of the generating functional,

$$
\int D[\Pi] e^{i S}=\int D\left[U^{\prime}\right] e^{-\int_{0}^{-i \beta} \int d^{d} x U^{\prime}(\mathbf{x}, t) \rho(\mathbf{x}, t)} Z\left(\left[U^{\prime}\right]\right)
$$

One may now use the above expression to relate the generating function of the free theory with that of the interacting theory. Thus,

$$
\begin{gathered}
Z([U])=\int D[\rho] \int D[\Pi] e^{i S_{\text {free }}} e^{-\frac{i}{2} \int_{0}^{-i \beta} d t \int d^{d} x \int d^{d} x^{\prime} V\left(\mathbf{x}-\mathbf{x}^{\prime}\right) \rho(\mathbf{x}, t) \rho\left(\mathbf{x}^{\prime}, t\right)} e^{\int_{0}^{-i \beta} \int d^{d} x U(\mathbf{x}, t) \rho(\mathbf{x}, t)} \\
=\int D\left[U^{\prime}\right] \int D[\rho] e^{-\frac{i}{2} \int_{0}^{-i \beta} d t \int d^{d} x \int d^{d} x^{\prime} V\left(\mathbf{x}-\mathbf{x}^{\prime}\right) \rho(\mathbf{x}, t) \rho\left(\mathbf{x}^{\prime}, t\right)} e^{\int_{0}^{-i \beta} \int d^{d} x\left(U(\mathbf{x}, t)-U^{\prime}(\mathbf{x}, t)\right) \rho(\mathbf{x}, t)} Z_{\text {free }}\left(\left[U^{\prime}\right]\right)
\end{gathered}
$$

We may now decompose the density variable into the various Fourier modes and perform the integration over the density variable and write,

$$
Z([U])=\int D\left[U^{\prime}\right] e^{\sum_{\mathbf{q} \neq 0 n} \frac{V}{2 \beta V_{\mathbf{q}}}\left(U_{\mathbf{q}^{n}}-U_{\mathbf{q}, n}^{\prime}\right)\left(U_{-\mathbf{q},-n}-U_{-\mathbf{q},-n}^{\prime}\right)} Z_{\text {free }}\left(\left[U^{\prime}\right]\right)
$$

where $V_{\mathbf{q}}=\int d^{d} x V(\mathbf{x}) e^{-i \mathbf{q} \cdot \mathbf{x}}$ and $U_{\mathbf{q} n}=\frac{1}{V} \int_{0}^{-i \beta} d t \int d^{d} x U(\mathbf{x}, t) e^{-i \mathbf{q} \cdot \mathbf{x}} e^{-z_{n} t}$. The generating functional of the free theory may be written as follows.

$$
Z_{\text {free }}([U])=e^{\frac{1}{2 !} \sum_{\mathbf{q} \neq 0, n}<\rho_{\mathbf{q} n} \rho_{-\mathbf{q},-n}>_{0} U_{\mathbf{q} n} U_{-\mathbf{q},-n}} e^{\frac{1}{3 !} \sum_{\mathbf{q} n ; \mathbf{q}^{\prime}, n^{\prime}}\left\langle\rho_{\mathbf{q} n} \rho_{\mathbf{q}^{\prime}, n^{\prime}} \rho_{-\mathbf{q}-\mathbf{q}^{\prime},-n-n^{\prime}}>_{0} U_{\mathbf{q} n} U_{\mathbf{q}^{\prime}, n^{\prime}} U_{-\mathbf{q}-\mathbf{q}^{\prime},-n-n^{\prime}} \ldots\right.}
$$

Thus we may rightly suspect that retaining only the gaussian terms in the above generating functional gives us the density-density correlation in the sense of RPA. Corrections to RPA are obtained by retaining the higher order terms involving three-body density correlations. Unfortunately, the current-current correlations are not so simple. This is because the current is a nonlinear combination of the density and conjugate variables. However, one may contemplate reverting to the Hamiltonian description and invoking the equation of continuity to relate the currentcurrent correlations with the density-density correlations.

Next we wish to show how to express the annihilation operator in momentum space $c_{\mathbf{p}}$ directly in terms of seabosons 7] in contrast to the exercise just completed, where we expressed only the slow part of the field in terms of the current and densities. To be sure, the exercise in this section is also valid only in the RPA-sense and hence only provides the asymptotics. Define $n_{\mathbf{q}}(\mathbf{k})=c_{\mathbf{k}-\mathbf{q} / 2}^{\dagger} c_{\mathbf{k}+\mathbf{q} / 2}$ for $\mathbf{q} \neq 0$. Hence we may write,

$$
\left[c_{\mathbf{p}}, n_{\mathbf{q}}(\mathbf{k})\right]=\delta_{\mathbf{p}, \mathbf{k}-\mathbf{q} / 2} c_{\mathbf{p}+\mathbf{q}} \equiv \delta_{\mathbf{p}, \mathbf{k}-\mathbf{q} / 2} T_{\mathbf{q}}(\mathbf{p}) c_{\mathbf{p}}
$$

where $T_{\mathbf{q}}(\mathbf{p}) \equiv \exp \left(\mathbf{q} \cdot \nabla_{\mathbf{p}}\right)$ is the translation operator. Also,

$$
\left[n_{\mathbf{q}}(\mathbf{k}), n_{-\mathbf{q}^{\prime}}\left(\mathbf{k}^{\prime}\right)\right]=\left[c_{\mathbf{k}-\mathbf{q} / 2}^{\dagger} c_{\mathbf{k}+\mathbf{q} / 2}, c_{\mathbf{k}^{\prime}+\mathbf{q}^{\prime} / 2}^{\dagger} c_{\mathbf{k}^{\prime}-\mathbf{q}^{\prime} / 2}\right] \approx\left(n_{F}(\mathbf{k}-\mathbf{q} / 2)-n_{F}(\mathbf{k}+\mathbf{q} / 2)\right) \delta_{\mathbf{k}, \mathbf{k}^{\prime}} \delta_{\mathbf{q}, \mathbf{q}^{\prime}}=\operatorname{sgn}(\mathbf{k} \cdot \mathbf{q}) \delta_{\mathbf{k}, \mathbf{k}^{\prime}} \delta_{\mathbf{q}, \mathbf{q}^{\prime}}
$$


The last two approximations are equivalent to the random phase approximation. The commutation rule in Eq.(33) may be exponentiated as follows,

$$
c_{\mathbf{p}}=e^{-\sum_{\mathbf{q}} \operatorname{sgn}\left(\mathbf{p} \cdot \mathbf{q}+\mathbf{q}^{2} / 2\right) n_{-\mathbf{q}}(\mathbf{p}+\mathbf{q} / 2) T_{\mathbf{q}}(\mathbf{p})} f(\mathbf{p})
$$

where $f(\mathbf{p})$ is an integration constant independent of $n_{\mathbf{q}}(\mathbf{k})$, the exact nature of which will be discussed subsequently. To be sure there are additional terms caused by the fact that the translational operator also acts on these commutators and so on. The reasons why these issues are not important are relegated to future publications. Suffice it to say that this is consistent with the RPA. Now we wish to evaluate $f(\mathbf{p})$. To this end we make the following surmise 7 ],

$$
f(\mathbf{p})=e^{-i P_{0}(\mathbf{p})} \sqrt{n_{0}(\mathbf{p})}
$$

Here $n_{0}(\mathbf{p})=c_{\mathbf{p}}^{\dagger} c_{\mathbf{p}}$ and $P_{0}(\mathbf{p})$ is canonically conjugate to $n_{0}(\mathbf{p})$, in other words, $\left[P_{0}(\mathbf{p}), n_{0}\left(\mathbf{p}^{\prime}\right)\right]=i \delta_{\mathbf{p}, \mathbf{p}^{\prime}}$. This ensures that $\left[c_{\mathbf{p}}, n_{0}\left(\mathbf{p}^{\prime}\right)\right]=c_{\mathbf{p}} \delta_{\mathbf{p}, \mathbf{p}^{\prime}}$. We have also set $\left[n_{\mathbf{q}}(\mathbf{k}), n_{0}(\mathbf{p})\right]=0$. In practical computations, we are going to set $n_{0}(\mathbf{p}) \approx n_{F}(\mathbf{p})=\theta\left(k_{F}-|\mathbf{p}|\right)$. This means we have to ensure that $P_{0}(\mathbf{p})$ is formally infinite in order for it to be a conjugate to $n_{0}(\mathbf{p})$. Thus we redefine,

$$
f(\mathbf{p})=e^{-i N^{0} \xi(\mathbf{p})} n_{F}(\mathbf{p})
$$

Here $N^{0}$ is a large quantity (total number of particles) and $\xi$ is an arbitrary c-number function. This means it obeys,

$$
L t_{N^{0} \rightarrow \infty} e^{-i N^{0} \xi(\mathbf{p})} e^{i N^{0} \xi\left(\mathbf{p}^{\prime}\right)}=\delta_{\mathbf{p}, \mathbf{p}^{\prime}}
$$

Unfortunately this prescription leaves out an essential part of the dynamics. For example we expect that for the free theory, $c_{\mathbf{p}}(t)=e^{-i \epsilon_{\mathbf{p}} t} c_{\mathbf{p}}(0)$. This is not obeyed unless we retain $P_{0}(\mathbf{p})$ as an operator conjugate to $n_{0}(\mathbf{p})^{2}$. Thus we will have to put the rapidly varying exponential in by hand during practical computations. More precisely, we are going to multiply and divide by the free propagator and use the bosonized version in the denominator and the one obtained from elementary considerations in the numerator thereby rendering this issue moot. In terms of bosonic operators defined in our earlier work $[\mathbf{7}], n_{\mathbf{q}}(\mathbf{k})=A_{\mathbf{k}}(\mathbf{q})+A_{\mathbf{k}}^{\dagger}(-\mathbf{q})$. Therefore, $\operatorname{sgn}\left(\mathbf{p} \cdot \mathbf{q}+\mathbf{q}^{2} / 2\right) n_{-\mathbf{q}}(\mathbf{p}+\mathbf{q} / 2)=-A_{\mathbf{p}+\mathbf{q} / 2}(-\mathbf{q})+A_{\mathbf{p}+\mathbf{q} / 2}^{\dagger}(\mathbf{q})$. In other words finally we obtain,

$$
c_{\mathbf{p}}=e^{\sum_{\mathbf{q} \neq 0}\left(A_{\mathbf{p}+\mathbf{q} / 2}(-\mathbf{q})-A_{\mathbf{p}+\mathbf{q} / 2}^{\dagger}(\mathbf{q})\right) T_{\mathbf{q}}(\mathbf{p})} e^{-i N^{0} \xi(\mathbf{p})} n_{F}(\mathbf{p})
$$

By expanding the exponential, it can be easily verified that this formula is consistent with the definition $n_{\mathbf{q}}(\mathbf{k})=$ $c_{\mathbf{k}-\mathbf{q} / 2}^{\dagger} c_{\mathbf{k}+\mathbf{q} / 2}$. Notice that there are no Klein factors in this formula either. Practical applications of the above formula will be relegated to future publications. One may contemplate using Eq.(39) to compute the one-particle Green function of any Fermi system. The results are valid at the level of RPA. In fact in an earlier work[4], one of the authors has highlighted the novel non-Fermi liquid character of a system described by long-range interactions in one dimension. In the following section, we study the same system in two spatial dimensions rather than in one. Here too we find that the system is an anomalous metal ( or an insulator ) but of the usual power-law Luttinger kind.

\section{METAL-INSULATOR QUANTUM PHASE TRANSITION IN TWO DIMENSIONS}

Now we wish to turn our attention to the properties of a system of spinless electrons mutually interacting via a gauge potential $v_{\mathbf{q}}=2 \pi e^{2} / q^{2}$ in two spatial dimensions. In real space this corresponds to a $\log (r)$ potential. Theories of novel non-Fermi liquid properties in higher dimensions are rare one such study for example is by Gori-Giorgi and Ziesche 13]. We consider the following hamiltonian in the sea-boson language [4].

$$
H=\sum_{\mathbf{k q}}\left(\frac{\mathbf{k} \cdot \mathbf{q}}{m}\right) A_{\mathbf{k}}^{\dagger}(\mathbf{q}) A_{\mathbf{k}}(\mathbf{q})+\sum_{\mathbf{q} \neq 0} \frac{v_{\mathbf{q}}}{2 V} \sum_{\mathbf{k k}^{\prime}}\left[A_{\mathbf{k}}(-\mathbf{q})+A_{\mathbf{k}}^{\dagger}(\mathbf{q})\right]\left[A_{\mathbf{k}^{\prime}}(\mathbf{q})+A_{\mathbf{k}^{\prime}}^{\dagger}(-\mathbf{q})\right]
$$

\footnotetext{
${ }^{2}$ Since $n_{\mathbf{q}}(\mathbf{k})$ varies slowly in time we may ignore it for this aspect of the discussion.
} 
This represents electrons mutually interacting via a two body potential $v_{\mathbf{q}}$ such that only small momentum transfer terms are important (forward scattering only) and $\mathbf{k}$ and $\mathbf{k}^{\prime}$ are close to the Fermi surface. Such a constraint is realized by the choice $v_{\mathbf{q}}=2 \pi e^{2} / q^{2}$ since the potential is singular for small $q$ and negligible for larger $q$. This hamiltonian may be easily diagonalized via a Bogoliubov transformation. We may borrow the results of Ando et al 14] for the dielectric function of a two dimensional electron gas to arrive at the following formula for the dispersion of collective modes : $\omega_{c}(q) \approx \frac{e k_{F}}{\sqrt{2 m}}+\left(\frac{3 k_{F}}{4 \sqrt{2} e m^{\frac{3}{2}}}\right) q^{2}$. The smallness of $q$ is governed by a cutoff $\Lambda$ which is the value of $q$ for which the second term is of the same order as the first. This means $\Lambda \approx \frac{2}{\sqrt{3}} e \sqrt{m}$. The momentum distribution of this system at zero temperature may be evaluated using the sea-boson formulas [4].

$$
<n_{\mathbf{k}}>=\frac{1}{2}\left[1+e^{-2 S_{B}^{0}(\mathbf{k})}\right] n_{F}(\mathbf{k})+\frac{1}{2}\left[1-e^{-2 S_{A}^{0}(\mathbf{k})}\right]\left(1-n_{F}(\mathbf{k})\right)
$$

where $S_{A}^{0}(\mathbf{k})=\sum_{\mathbf{q}}<A_{\mathbf{k}-\mathbf{q} / 2}^{\dagger}(\mathbf{q}) A_{\mathbf{k}-\mathbf{q} / 2}(\mathbf{q})>$ and $S_{B}^{0}(\mathbf{k})=\sum_{\mathbf{q}}<A_{\mathbf{k}+\mathbf{q} / 2}^{\dagger}(\mathbf{q}) A_{\mathbf{k}+\mathbf{q} / 2}(\mathbf{q})>$ and $n_{F}(\mathbf{k})=\theta\left(k_{F}-|\mathbf{k}|\right)$. In the $q$ summation we retain only the most singular parts and integrate only upto the cutoff $\Lambda$. We omit the rest of the details regarding computation of the boson occupations. When this is done we obtain the following formula for the momentum distribution.

$$
<n_{\mathbf{k}}>=\frac{1}{2}+\frac{1}{2}\left(\frac{\left|k_{F}-\right| \mathbf{k}||}{\Lambda^{\prime}}\right)^{\gamma} \operatorname{sgn}\left(k_{F}-|\mathbf{k}|\right)
$$

where $\gamma=\frac{m}{k_{F}} \frac{e}{\sqrt{2 m}}=\frac{\omega_{0}}{2 E_{F}}$ and $\Lambda^{\prime}=e^{-\frac{2}{\pi}} \Lambda$ and $\omega_{0}=\frac{e k_{F}}{\sqrt{2 m}}$. Thus the system an insulator of the usual Luttinger type for $\omega_{0}>2 E_{F}$ and an anomalous metal with a residual Fermi surface for $\omega_{0}<2 E_{F}$. We may now compute the dynamical Green function using the methods outlined in the earlier sections, but this is not going to yield any new information, since the important attributes namely the estimate of the plasmon energy and the anomalous exponent have already been highlighted. It would also be interesting to see if the usual Coulomb interaction in three spatial dimensions also exhibits some kind of metal insulator transition at absolute zero temperature as a function of density. Unfortunately in order to study this reliably we have to go beyond the RPA and this calculation is beyond the scope of this preliminary work that focuses mainly on formalism.

\section{CONCLUSIONS}

To conclude, we have shown that it is possible to bosonize fermions in arbitrary dimensions and relate bosonization with quantum hydrodynamics. We have been able to extract the dynamical one-particle Green function in terms of the current and density correlation functions valid at the level of RPA. We have expressed the fermion annihilation operator in momentum space directly in terms of bosonic operators called sea-bosons. We have also derived a general formula for the generating function of density correlations of systems interacting via two-body forces in terms of the corresponding quantity for the free theory. Finally we have evaluated the momentum distribution of a two-dimensional anomalous metal ( or insulator ) with the electrons interacting via a long range potential and extracted the anomalous exponent.

\section{APPENDIX}

The connection between the current-current correlations and density-density correlation implied by the equation of continuity is recovered by the action Eq. 25) postulated in the main text. This may be verified at the level of RPA in any number of dimensions as follows. Define,

$$
\begin{gathered}
Z_{d}[U] \equiv \int D[\rho] D[\Pi] e^{i \int\left(\rho \partial_{t} \Pi-\rho \partial_{t} \Lambda-\frac{\rho(\nabla \Pi)^{2}+\frac{(\nabla \rho)^{2}}{4 \rho}}{2 m}\right)}+\int U \rho \\
Z_{c}[V] \equiv \int D[\rho] D[\Pi] e^{i \int\left(\rho \partial_{t} \Pi-\rho \partial_{t} \Lambda-\frac{\rho(\nabla \Pi)^{2}+\frac{(\nabla \rho)^{2}}{4 \rho}}{2 m}\right)}-\int \mathbf{J} \cdot \nabla V
\end{gathered}
$$


Then $<T\left[\rho(\mathbf{x}, t) \rho\left(\mathbf{x}^{\prime}, t^{\prime}\right)\right]>=\frac{\delta^{2} Z_{d}[U]}{\delta U(\mathbf{x}, t) \delta U\left(\mathbf{x}^{\prime}, t^{\prime}\right)}$ and $<T\left[\left(\nabla_{\mathbf{x}} \cdot \mathbf{J}(\mathbf{x}, t)\right)\left(\nabla_{\mathbf{x}^{\prime}} \cdot \mathbf{J}\left(\mathbf{x}^{\prime}, t^{\prime}\right)\right)\right]>=\frac{\delta^{2} Z_{c}[V]}{\delta V(\mathbf{x}, t) \delta V\left(\mathbf{x}^{\prime}, t^{\prime}\right)}$. We may relate $Z_{c}$ and $Z_{d}$ as follows.

$$
Z_{c}[V]=Z_{d}\left[-\frac{i m}{2}(\nabla V)^{2}+m \partial_{t} V\right]
$$

The validity of this may be verified at the level of RPA. At this level we may write,

$$
Z_{d}[U]=e^{\frac{1}{2} \int<T\left[\rho(\mathbf{x}, t) \rho\left(\mathbf{x}^{\prime}, t^{\prime}\right)\right]>U(\mathbf{x}, t) U\left(\mathbf{x}^{\prime}, t^{\prime}\right)}
$$

This implies,

$$
\begin{gathered}
<T\left[\nabla_{\mathbf{x}} \cdot \mathbf{J}(\mathbf{x}, t) \nabla_{\mathbf{x}^{\prime}} \cdot \mathbf{J}\left(\mathbf{x}^{\prime}, t^{\prime}\right)\right]>=\int<T\left[\rho(\mathbf{y}, T) \rho\left(\mathbf{y}^{\prime}, T^{\prime}\right)\right]>\left(m \delta(\mathbf{y}-\mathbf{x}) \partial_{T} \delta(T-t)\right)\left(m \delta\left(\mathbf{y}^{\prime}-\mathbf{x}^{\prime}\right) \partial_{T^{\prime}} \delta\left(T^{\prime}-t^{\prime}\right)\right) \\
=m^{2}<T\left[\partial_{t} \rho(\mathbf{x}, t) \partial_{t^{\prime}} \rho\left(\mathbf{x}^{\prime}, t^{\prime}\right)\right]>
\end{gathered}
$$

The validity of the above equation follows from the equation of continuity.

\section{DIRECTOR'S CUT}

In this section, we provide a new proof of the irrotational nature of the velocity operator for fermions. This makes the above paper logically complete. Unfortunately this proof is not there in the published version.

Lemma: If $\nabla \rho \times \mathbf{J}-\rho \nabla \times \mathbf{J}=0$ then there exists a $\Pi$ such that $\mathbf{J}=-\rho \nabla \Pi$.

Proof: First we note that $[\mathbf{J}(\mathbf{x}), \rho(\mathbf{x})]=0$, hence we may write $\frac{\mathbf{J}}{\rho}$ without ambiguity.

$$
\nabla \times \frac{\mathbf{J}}{\rho}=\frac{1}{\rho} \nabla \times \mathbf{J}-\frac{1}{\rho^{2}} \nabla \rho \times \mathbf{J}=\frac{1}{\rho^{2}}[\rho \nabla \times \mathbf{J}-\nabla \rho \times \mathbf{J}]=0
$$

Hence $\frac{\mathbf{J}}{\rho}=-\nabla \Pi$ for some $\Pi$. Hence $\mathbf{J}=-\rho \nabla \Pi$. Now we prove that $\nabla \rho \times \mathbf{J}-\rho \nabla \times \mathbf{J}=0$ for (free) fermions. We do this using the first quantized definitions of current and density operator and using real space fermion wavefunctions.

$$
\begin{gathered}
\rho(\vec{r})=\sum_{j=1}^{N} \delta\left(\vec{r}-\vec{r}_{j}\right) \\
\mathbf{J}(\vec{r})=\frac{i}{2} \sum_{j=1}^{N}\left[\nabla \delta\left(\vec{r}-\vec{r}_{j}\right)\right]-i \sum_{j=1}^{N} \delta\left(\vec{r}-\vec{r}_{j}\right) \nabla_{j} \\
\nabla \rho(\vec{r}) \times \mathbf{J}(\vec{r})=\sum_{j=1}^{N}\left[\nabla \delta\left(\vec{r}-\vec{r}_{j}\right)\right] \times \frac{i}{2} \sum_{p=1}^{N}\left[\nabla \delta\left(\vec{r}-\vec{r}_{p}\right)\right]-\sum_{j=1}^{N}\left[\nabla \delta\left(\vec{r}-\vec{r}_{j}\right)\right] \times i \sum_{p=1}^{N} \delta\left(\vec{r}-\vec{r}_{p}\right) \nabla_{p} \\
\rho(\vec{r}) \nabla \times \mathbf{J}(\vec{r})=-i \sum_{j=1}^{N} \delta\left(\vec{r}-\vec{r}_{j}\right) \sum_{p=1}^{N}\left[\nabla \delta\left(\vec{r}-\vec{r}_{p}\right)\right] \times \nabla_{p} \\
\mathbf{V}(\vec{r})=\sum_{j, p=1}^{N}\left[\nabla \delta\left(\vec{r}-\vec{r}_{j}\right)\right] \times \frac{i}{2}\left[\nabla \delta\left(\vec{r}-\vec{r}_{p}\right)\right]-i \sum_{j, p=1}^{N} \delta\left(\vec{r}-\vec{r}_{p}\right)\left[\nabla \delta\left(\vec{r}-\vec{r}_{j}\right)\right] \times \nabla_{p}+i \sum_{j, p=1}^{N} \delta\left(\vec{r}-\vec{r}_{j}\right)\left[\nabla \delta\left(\vec{r}-\vec{r}_{p}\right)\right] \times \nabla_{p}
\end{gathered}
$$




$$
\mathbf{V}(\vec{r})=\frac{i}{2} \sum_{j, p=1}^{N}\left(\delta\left(\vec{r}-\vec{r}_{j}\right)\left[\nabla \delta\left(\vec{r}-\vec{r}_{p}\right)\right]-\delta\left(\vec{r}-\vec{r}_{p}\right)\left[\nabla \delta\left(\vec{r}-\vec{r}_{j}\right)\right]\right) \times\left(\nabla_{p}-\nabla_{j}\right)
$$

Let, $C\left(\vec{r}_{j}, \vec{r}_{p}\right) \equiv\left(\delta\left(\vec{r}-\vec{r}_{j}\right)\left[\nabla \delta\left(\vec{r}-\vec{r}_{p}\right)\right]-\delta\left(\vec{r}-\vec{r}_{p}\right)\left[\nabla \delta\left(\vec{r}-\vec{r}_{j}\right)\right]\right) \times\left(\nabla_{p}-\nabla_{j}\right)$ and $f\left(\vec{r}_{j}, \vec{r}_{p}\right)$ be some function :

$$
\begin{gathered}
\int d \vec{r}_{j} \int d \vec{r}_{p} C\left(\vec{r}_{j}, \vec{r}_{p}\right) f\left(\vec{r}_{j}, \vec{r}_{p}\right)=\int d \vec{r}_{j} \int d \vec{r}_{p}\left(\delta\left(\vec{r}-\vec{r}_{j}\right)\left[\nabla \delta\left(\vec{r}-\vec{r}_{p}\right)\right]-\delta\left(\vec{r}-\vec{r}_{p}\right)\left[\nabla \delta\left(\vec{r}-\vec{r}_{j}\right)\right]\right) \times\left(\vec{f}_{2}\left(\vec{r}_{j}, \vec{r}_{p}\right)-\vec{f}_{1}\left(\vec{r}_{j}, \vec{r}_{p}\right)\right) \\
=\left(\int d \vec{r}_{p}\left[\nabla \delta\left(\vec{r}-\vec{r}_{p}\right)\right] \times \vec{f}_{2}\left(\vec{r}, \vec{r}_{p}\right)-\int d \vec{r}_{j}\left[\nabla \delta\left(\vec{r}-\vec{r}_{j}\right)\right] \times \vec{f}_{2}\left(\vec{r}_{j}, \vec{r}\right)\right) \\
-\left(\int d \vec{r}_{p}\left[\nabla \delta\left(\vec{r}-\vec{r}_{p}\right)\right] \times \vec{f}_{1}\left(\vec{r}, \vec{r}_{p}\right)-\int d \vec{r}_{j}\left[\nabla \delta\left(\vec{r}-\vec{r}_{j}\right)\right] \times \vec{f}_{1}\left(\vec{r}_{j}, \vec{r}\right)\right) \\
=\sum_{I} \hat{e}_{I} \epsilon_{I J K}\left(\vec{f}_{2, J ; 2, K}(\vec{r}, \vec{r})-\vec{f}_{1, J ; 2, K}(\vec{r}, \vec{r})\right)-\sum_{I} \hat{e}_{I} \epsilon_{I J K}\left(\vec{f}_{2, J ; 1, K}(\vec{r}, \vec{r})-\vec{f}_{1, J ; 1, K}(\vec{r}, \vec{r})\right) \\
f_{1, J ; 2, K}(\vec{r}, \vec{r})=\left[\frac{\partial}{\partial x_{J}} \frac{\partial}{\partial y_{K}} f(\mathbf{X}, \mathbf{Y})\right]_{\mathbf{X}=\mathbf{Y}=\vec{r}}=s\left[\frac{\partial}{\partial x_{J}} \frac{\partial}{\partial y_{K}} f(\mathbf{Y}, \mathbf{X})\right]_{\mathbf{X}=\mathbf{Y}=\vec{r}}=s f_{2, J ; 1, K}(\vec{r}, \vec{r}) \\
f_{2, J ; 2, K}(\vec{r}, \vec{r})=\left[\frac{\partial}{\partial y_{J}} \frac{\partial}{\partial y_{K}} f(\mathbf{X}, \mathbf{Y})\right]_{\mathbf{X}=\mathbf{Y}=\vec{r}}=s\left[\frac{\partial}{\partial y_{J}} \frac{\partial}{\partial y_{K}} f(\mathbf{Y}, \mathbf{X})\right]_{\mathbf{X}=\mathbf{Y}=\vec{r}}=s f_{1, J ; 1, K}(\vec{r}, \vec{r}) \\
\ldots=\left(f_{2, J ; 2, K}(\vec{r}, \vec{r})-f_{1, J ; 2, K}(\vec{r}, \vec{r})\right)-\left(f_{2, J ; 1, K}(\vec{r}, \vec{r})-f_{1, J ; 1, K}(\vec{r}, \vec{r})\right) \\
=(s+1) f_{1, J ; 1, K}(\vec{r}, \vec{r})-(s+1) f_{2, J ; 1, K}(\vec{r}, \vec{r})
\end{gathered}
$$

For $s=-1$ then these terms are zero.

\section{Acknowledgments}

The author would like to thank S. Basu for taking active interest in the progress of this work and for various useful remarks that enhanced the content of the work. It is also a pleasure to acknowledge discussions with S. Das and S. Rao while one of the authors (G.S.S.) was at the Harish Chandra Research Institute. An early draft of this article was completed at this Institute.

[1] J. Maes, M. Hayne, Y. Sidor, B. Partoens, F. M. Peeters, Y. Gonzlez, L. Gonzlez, D. Fuster, J. M. Garca, and V. V. Moshchalkov Phys. Rev. B, 70, 155311 (2004); S. J. Tans, M. H. Devoret, H. Dai, A. Thess, R. E. Smalley, L. J. Geerligs, and C. Dekker, Nature, 386, 474 (1997) ; M. Bockrath, D. H. Cobden, P. L. McEuen, N. G. Chopra, A. Zettl, A. Thess, and R. E. Smalley, Science, 275, 1922 (1997); K.J. Thomas, J.T. Nicholls, M. Pepper, W.R. Tribe, M.Y. Simmons and D.A. Ritchie, Phys. Rev. B 61, R13 365 (2000).

[2] S. Lal, S. Rao and D. Sen, Phys. Rev. Lett., 87, 026801 (2001); Phys. Rev. B 66, 165327 (2002); S. Das and S. Rao, Phys. Rev. B 70, 155420 (2004).

[3] F.D.M. Haldane, Phys. Rev. Lett. 45, 1358 (1980); Phys. Rev. Lett. 47, 1840 (1981); J. Phys. C 14, 2585 (1981); Helv. Phys. Acta 65, 152 (1992); Perspectives in Many-Particle Physics, Proc. of the Intl. School of Physics "Enrico Fermi" Course CXXI, Varenna, 1992 eds. R. Schreiffer and R. A. Broglia ( North Holland, New York, 1994 ); A. Luther, Phys. Rev. B 19, 320 (1979). 
[4] G.S. Setlur, Pramana, 62, 115 (2004).

[5] G.S. Setlur and Y.C. Chang, Phys. Rev. B, 57, 15144 (1998).

[6] C. Chamon, M. Oshikawa and I. Affleck, Phys. Rev. Lett. 91, 206403 (2003).

[7] G.S. Setlur and D.S. Citrin, Phys. Rev. B, 65, 165111 (2002).

[8] See the article by R. Shankar in Condensed Matter and Particle Physics, eds. Y. Lu, J. Pati and Q. Shafi, World Scientific, 1993.

[9] J. von Delft and H. Schoeller, Ann. der Physik 7, 225 (1998), see also cond-mat/9805275 Field Theories in Condensed Matter Physics, Hindustan Book Agency, ed. Sumathi Rao (c)2001.

[10] G.S. Setlur, Pramana, 62, 101 (2004).

[11] K. Schotte and U. Schotte, Phys. Rev. 182, 479 (1969).

[12] A.K. Rajagopal and G.S. Grest, Phys. Rev. A 10, 1837 (1974); Phys. Rev. A 10, 1395 (1974); Prog. Theor. Phys. 52, 811 (1974); 52, 1719 (1974) Errata, A. K. Rajagopal, in Quantum Fluids, in particular, superfluid helium, Three Lectures in the Bose Symposiums on Statistical Physics (July 15-27, 1974). Supplement to J. Ind. Inst. Sci. 85, (1975).

[13] P. Gori-Giorgi and P. Ziesche, Phys. Rev. B 66, 235116 (2002); also as cond-mat/0205342

[14] T. Ando, A. B. Fowler and F. Stern, Rev. Mod. Phys. 54, 437 (1982). 